\title{
Simulation of the process and system of power plants exhaust gases purification during construction and re-construction of housing and utilities infrastructure of urban areas
}

\author{
Ekaterina Lysova ${ }^{1, *}$, Oksana Paramonova ${ }^{1}$, and Oksana Gurova ${ }^{1}$ \\ ${ }^{1}$ Department of Environmental Engineering, Federal State-Funded Educational Institution of Higher Education Don State Technical \\ University, Rostov-on-Don, Russian Federation
}

\begin{abstract}
Urban territories shall provide for favorable life activity of people alongside with preservation of ecological balance, however, they are peculiar city-planning "focuses" on which ecological problems are maximally concentrated. Air basin of urban territories is the most dynamic and most vulnerable of all the components of environment exposed to maximal anthropogenic load. The solution of the actual problem of increase of ecological safety of urban territories on basis of imitational simulation of the process and system of purification of exhaust gases of power plants of housing and utilities infrastructure with the help of management of parameters substantially influencing the ecological efficiency and energy intensity value of the purification process is represented in the article. Management of parameters is possible on the stage of designing of system for purification of exhaust gases of power plants, which substantially optimizes the choice of ways and means for decrease of air media of urban territories.
\end{abstract}

\section{Introduction}

Under the modern conditions of development of urban territories the problem of pollution of air basin is rather pressing, because the air basin is subject to maximum anthropogenic load and is the most dynamic of all components of the environment [1-3]. Production of heat and electrical energy by power plants is accompanied with substantial environment pollution [4]. However, power plants are an important object of housing and utilities infrastructure of urban media and a necessary element of the system of life support of urban territories. They cannot be located outside the urban territories which is related with minimization of losses, both material and energetic [5].

Main contribution into the pollution is made by exhaust gases of power plants with which polluting substances come into the air basin. Structure of exhaust gases and amount of discharged polluting substances depends on the type of burnt fuel [6].

\section{Relevance, scientific importance of the issue with brief literature review}

Relevance of the article is explained by constant increase of air basin pollution, which is a problem of the majority of big cities and is related to the necessity of provision of ecological safety of urban territories, creation of favorable conditions for life of the population, and environmental protection.

Notwithstanding the sustainable trend of decrease of the volume and change of the structure of exhausts of polluting agents into the air basin during fuel burning, power plants are still taking one of the leading positions by contribution to atmospheric air pollution.

Works of such authors, as Belik S.E., Bespalov V.I., Boguslavskiy E.I., Valdberg A. Yu., Gibbs V., Gluzberg V. E., Greene X., Zhuravlev V. P., Zhuravlyov V. P., Ishchuk I. G., Kouzov P. A., Lane V., Livchak I.F., Logachev I. N., Minko V. A., Mishner Y, PetryanovSokolov I. V., Rehbinder P. A., Rekun V. V., Rusanov A.A, Saranchuk V. I., Segal I. Ya., Sidorenko V. F., Sou S., Spurny To., Sutugin A. G., Uzhov V. N., Fuchs N. A., Shtokman E. A., Strauss W. and many other authors are dedicated to research and evaluation of negative impact of power plants on the environmental components, studying of characteristics of polluting aerosol, including as a disperse system, its behavior under the conditions of external impact, examination of series of tasks related to purification, as well as improvement of technique of air purification [7-12].

However, it is important to make a right choice of constructive elements and working characteristics of the process and system of exhaust gases purification on the designing stage. It will provide for maximum ecological efficiency and energy economic feasibility of the process.

Thus, relevance of research aimed at simulation of the process and system of purification of exhaust gases of power plants of housing and utilities infrastructure and management of parameters influencing efficiency and energy intensity of the purification process does not cause any doubts.

\footnotetext{
* Corresponding author: katerina.lysova0803@gmail.com
} 


\section{Task setting}

To choose optimal process and system of exhaust gases purification of power plants, including on the designing stage, it is necessary to provide for possibility of quality process modelling. Solution of this task supposes construction of a physical model of decrease of pollution of air media for the conditions of operation of power plants and obtaining of parametric dependencies of efficiency and energy intensity value as the resulting parameters of purification process.

\section{Theoretical part}

Based on earlier carried out physical simulation of the process of pollution of air media [13] we have constructed a physical model of the process of decrease of air media pollution [14], which illustrates consecutive deliberate impact on polluting aerosol with external disperse systems on each stage of pollution process.

Decrease of concentration of polluting agents (PA) contained in exhaust gases of power plants could be achieved by realization of two main cycles of the process [14]:

1 - decrease of pollution of technological raw materials (different types of organic fuel) or technological equipment (boiler units) with the aim of prevention of exhaust of polluting agents particles from the volume of technological raw materials.

2 - decrease of pollution of air media with additional implementation on the operating or designed manufacturing and technological equipment (boiler unit) of special engineering and technical events (systems) with the aim of destruction of aerosol polluting agents at the account of division of disperse phase and dispersing medium.

First cycle of the process for power plants is more rational [15-17] and could include recirculation of exhaust gases, feeding of excess air amount into the burning zone, fuel desulfurization, additional of chemical substances, moisture sprinkling or steam into the furnace, preliminary fuel heating, etc. However, its use is economically unjustified, and main obstruction is manufacturing and technological requirements which contain limitations on parameters of characteristics of additional disperse systems.

With the purpose of decrease of air basin pollution of urban territories by power plants the second cycle of the process is used. During organic fuel burning there is no need in organization of the stage of pollutants collection, since they from the source of generation come into duct works. Thus, in our case, the second cycle of the process of decrease of air medium pollution includes implementation of the process of purification and dispersion (fig. 1).

Interconnection of parameters of external influences and gas aerosol is reflected in the physical model of the process of decrease of air medium pollution (fig. 1) through changes in the properties parameters (PP), energy parameters $\left(\mathrm{W}_{\mathrm{S}}\right)$ and persistence $(\mathrm{U})$ of interacting systems.
In the process of organic fuel burning at the power plants aerosol is created which lowers its persistence and transforms into the «Initial-I» («I-I») disperse system. Its persistence increases, since in the process of burning energy is constantly fed into this system.

In the process of purification disperse system «I-I» interacts with preliminary prepared «Additional-II.1» («A-II.1») disperse system. As the result of this interaction two disperse systems are created:

1 - «Residual-II.2» («R-II.2») disperse system, which is characterized by increased content of PA particles and increased persistence. «R-II.2» disperse system goes into the utilization system.

2 - «Intermediate-II.1» («In-II.1») disperse system polluting aerosol with certain residual concentration of polluting agents, directed either into the atmosphere (with adherence to maximum allowable emission), or into the zone of forced dispersion. At that «In-II.1» disperse system is characterized by increased value of persistence as an aerosol.

In the process of forced dispersion the disperse system «In-II.1» is purposefully influence by «Additional-II.3» («A-II.3») disperse system, preliminary prepared by parameters. As the result two disperse systems are created in aerosol state:

1 - «Residual-II.3» («R-II.3») disperse system represented by maximum number of polluting agents particles. After interaction with «A-II.3» disperse system it lowers its persistence as a polluting aerosol and is trying to transfer into the state of polluting agents material with increase, and then stabilization of its persistence.

2 - «Residual-II.4» («R-II.4») disperse system represented by minimal amount of polluting agents, it stays in the aerosol state, its persistence in time slightly grows, and then stabilizes. This system forms residual concentration of polluting agents in air basin of the reviewed territory.

As the result of consecutive impact on «Initial», «Intermediate» and «Residual» disperse systems with external additional disperse systems in accordance with laws of mass and energy preservation, transformation of interacting systems happens. This transformation consists of the following: newly created disperse systems are distinguished with properties parameters, energy parameters and persistence from the interacting ones.

From the position of the theory of persistence of disperse systems the essence of the process of decrease of air medium pollution lies in destruction of a disperse system - polluting agents being in different aggregate states. Mathematically the essence of the process of decrease of air medium pollution taking into consideration probability of implementation of each of its stages as dependent events in our cases is expressed by the formula $[14,18,19]$ : 


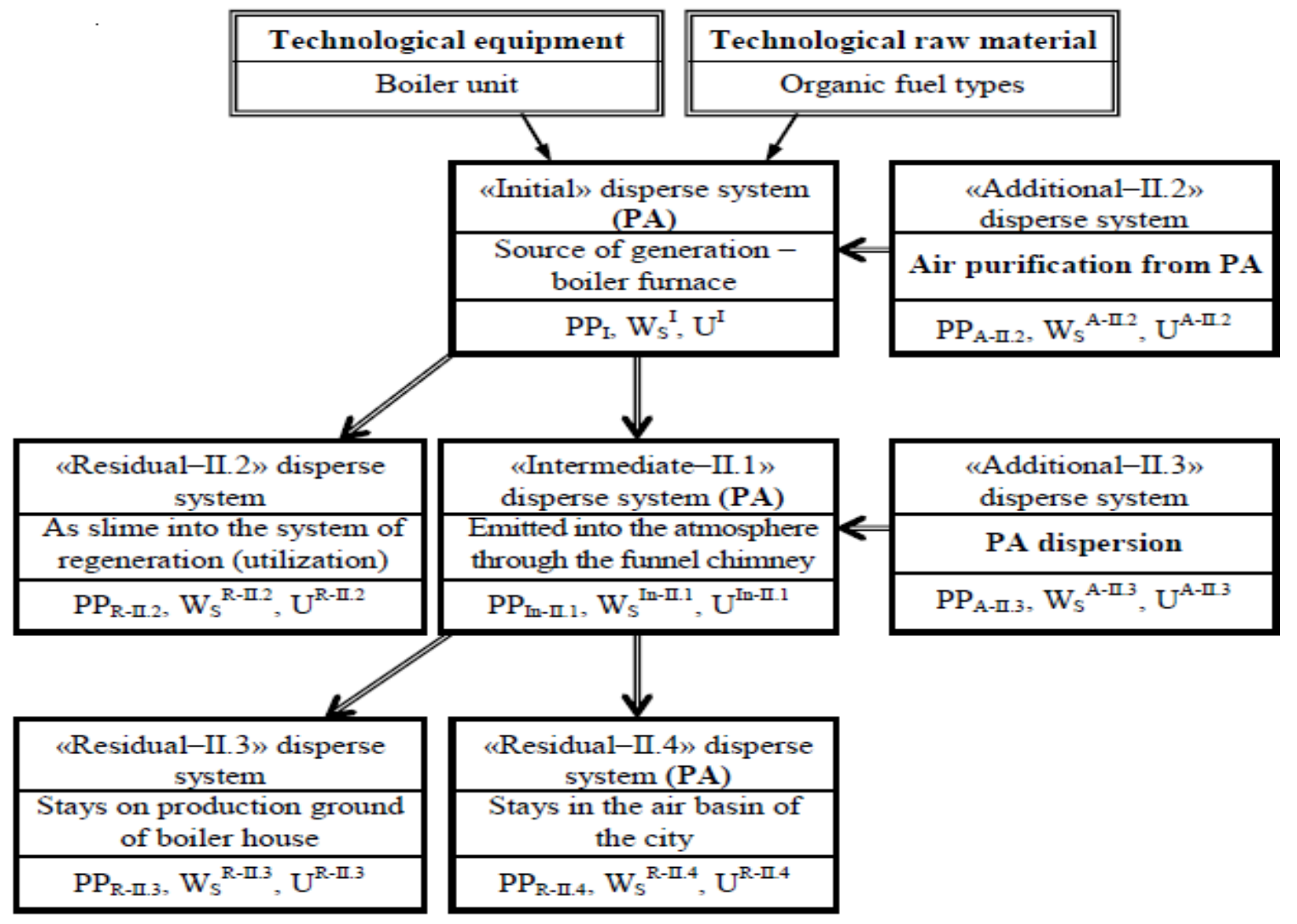

Fig. 1. Flow chart of the physical model of the process if decrease of air medium pollution for power plants

$$
P_{D A M P}=1-\left(1-P_{P P}\right) \cdot\left(1-P_{D P}\right),
$$

where $P_{P P}$ - probable implementation of the purification process; $P_{D P}-$ probability of implementation of dispersion process.

The created physical model of air medium pollution allows to suggest and simulate the system of purification in which on components of the exhaust gases shall be consecutively and purposefully influenced by corresponding external impacts.

In accordance with the suggested classification scheme of systems of control of polluting agents [20] for air purification from polluting agents contained in exhaust gases of power plants the following main methods are used [21]:

- hydrodynamic method (use of fluid as external impact managing the persistence of gas and solid PA contained in exhaust gases);

- aerodynamic method (use of gas (air) flows);

- electromagnetic method (use of electromagnetic field);

- thermophysical method (use of thermal field).

Out of known and traditionally applied methods for purification of exhaust gases of power plants we have chosen hydrodynamic method as one of the most widely spread and being rather highly ecologically efficient. Implementation of hydrodynamic method of purification of exhaust gases from gaseous matter is carried out by the following methods (and technical devices):

- use of steam, fog (steam chambers, for generators);

- use of foam (foam generators);
- by barbotage through a liquid layer (various types of barboters);

- by surface contact with a static layer of liquid;

- by irrigation with dispersed liquid (low-pressure, high-pressure, pneumohydroirrigation, irrigated gas ducts);

- by combination of listed methods (scrubbers, wet cyclones) under this method.

Determination of optimal working parameters of the process of hydrodynamic purification of exhaust gases, improvement of the process, simulation and management of the process are based on analysis of parametric dependencies of its resulting parameters - ecological efficiency and energy intensity value [22]. These criteria, in our opinion, are complementary and characterize the process of decrease of air basin most fully.

We understand the term «ecological efficiency» as the degree of decrease of concentration of polluting agents in the air basin at the account of implementation of the process of purification related to the concentration of polluting agents going into the air basin without purification.

To determine efficiency of purification of exhaust gases with hydroirrigation we have used probabilistic approach taking into consideration consecutive implementation of four stages of the process of interaction of the system «gas-fluid» both between each other and with the environment. At that the efficiency of this or that process is understood as the probability of its implementation.

The process of hydrodynamic purification includes 4 stages: 
1 - approximation of gas molecule with a drop to the distance when intermolecular forces of molecule interaction of gas and fluid start acting, at that, in our opinion, the underlying are turbulent and diffusion forces;

2 - transfer of gas molecule into the area on the boundary of phases division where, in our opinion, the main influence is carried out by absorption and molecular forces.

3 - penetration of the molecule into the volume of fluid where, in our opinion, it is necessary to take into account diffusion forces and chemical reactions between molecules of gas and fluid;

4 - settling of drops with molecules diluted in them from gas-air stream due to gravitation forces.

The term «energy efficiency» we understand as the share of energy used for achievement of the aim of the process of decrease of air medium pollution (useful energy) relative to the energy used for implementation of the process in general.

We understand useful energy as the energy used for interaction of gas stream with dispersed fluid:

- kinetic energy of fluid drops, into the boundary layer of which the gas molecules have diffused;

- kinetic energy of gas aerosol;

- energy of absorption interaction of gas and fluid molecules;

- energy of chemical interaction of gas molecules and irrigating fluid.

We consider as consumed the energy for irrigation:

- energy parameter of draft agitator characterizing energy consumed for organization of air flow with certain characteristics;

- energy parameter of pumping station characterizing energy consumed for organization of liquid flow with certain characteristics.

\section{Practical importance, suggestions and results of implementation, results of experimental research}

To simulate the process and system of purification of exhaust gases of power plants of housing and utility sector it is necessary in each certain case of their practical application to determine corresponding resulting parameters, which include, first of all, ecological efficiency and energy intensity value. Examination of physical essence of the process of hydrodynamic purification of exhaust gases allowed to carry out mathematical description of these resulting parameters.

Parametrical dependence of ecological efficiency of the process of gas purification with hydroirrigation is written as:

$$
\begin{gathered}
E_{e f}^{\text {hir }}=\left[1-\left(1-\frac{2}{3} \cdot \frac{1,8 B}{\mu_{a} \cdot v_{d} \cdot D_{g} \cdot d_{d}^{2}}\right)^{2 / 3} x\right. \\
\left.x\left(1-0,032 \cdot q \cdot \sqrt{\frac{d_{d} \cdot \rho_{l} \cdot \sigma_{l-g}}{Q_{l} \cdot D_{g} \cdot \mu_{a} \cdot v_{d}}}\right)\right] x \\
x\left(1-\frac{\rho_{l} \cdot d_{d}^{2} \cdot v_{d} \cdot e^{\alpha} \cdot E_{\mathrm{a}} \cdot \chi}{\frac{\rho_{l} \cdot d_{d}^{2} \cdot v_{d}}{36 \mu_{a} \cdot d_{d}}+10^{A n_{1}}}\right)
\end{gathered}
$$

where $\boldsymbol{B}$ - the constant of intermolecular interaction, $\mathrm{J} \cdot \mathrm{m}^{2} ; \boldsymbol{\mu}_{\boldsymbol{a}}-$ dynamic air viscosity, $\mathrm{Pa} \cdot \mathrm{s} ; \boldsymbol{v}_{\boldsymbol{d}}-$ mean drop speed, $\mathrm{m} / \mathrm{s} ; \boldsymbol{D}_{\boldsymbol{g}}$ - the coefficient of gas diffusion, $\mathrm{m}^{3} / \mathrm{s} ; \boldsymbol{d}_{\boldsymbol{d}}$ - the mean surface-bulk drop diameter, $\mathrm{m} ; \boldsymbol{q}$ - the electrical charge of liquid aerosol, $\mathrm{Kl} / \mathrm{kg} ; \boldsymbol{\rho} \boldsymbol{l}$ - the density of irrigating liquid, $\mathrm{kg} / \mathrm{m}^{3} ; \sigma_{l-g}-$ the superficial tension of fluid on the border «liquid-gas», N/m; $\boldsymbol{Q}_{\boldsymbol{l}}$ - the irrigating liquid consumption, $\mathrm{m}^{3} / \mathrm{c} ; \boldsymbol{\alpha}-$ the coefficient of absorption for gases and water; $\boldsymbol{E}_{\boldsymbol{a}}-$ the activation energy, $\mathrm{kJ} / \mathrm{mole} ; \chi$ - the absorption acceleration in the liquid phase during chemical reaction in it; $\boldsymbol{A}$ - the coefficient of fullness of ductwork closure with the flame body of dispersed fluid; $\boldsymbol{n}_{1}$ - the coefficient characterizing the speed mode of fluid aerosol distribution in the active purification zone.

Parametric dependence of energy intensive value of the process of hydrodynamic purification is written as:

$$
\begin{gathered}
E_{\text {hir }}^{E}=\frac{E_{e f}^{h i r} \cdot v_{d} \cdot h_{a} \cdot B_{1}}{9,8 H_{H}+\frac{2800 \cdot\left(H_{n m}-H_{n}\right)^{1,5} \cdot d_{a}^{2}}{\mu \pi \cdot d_{n}^{2} \sqrt{H_{H} \cdot g}} x} \\
x\left(0,5 \rho_{l}+\frac{10^{-26}}{g \mu_{a} \cdot d_{d}}+\frac{6 e^{\alpha} \cdot \sigma_{l-g} \cdot(1-\cos \chi)}{v_{d}^{2}}\right)
\end{gathered}
$$

where $\boldsymbol{h}_{\boldsymbol{a}}$ - the length of active zone of irrigation flame, $\mathrm{m} ; \boldsymbol{B}_{\boldsymbol{1}}-$ the coefficient taking into account direction and value of the speed of stream of polluting agents in the active zone of the purification process; $\boldsymbol{H}_{\boldsymbol{H}}$ - the pressure of fluid created by the pump directly in front of the irrigator, $\mathrm{Pa} ; \boldsymbol{H}_{\boldsymbol{n} \boldsymbol{m}}$ - the full pressure of air in the sucking in cross-section of draught activator (ventilator), $\mathrm{Pa} ; \boldsymbol{H}_{\boldsymbol{n}}$ - loss of pressure in the network of ducts from the sucking in cross-section of draught activator (ventilator) to the initial border of active zone of the purification process, $\mathrm{Pa} ; \boldsymbol{d}_{\boldsymbol{a}}$ - the equivalent diameter of cross-section of active zone of the purification process, $\mathrm{m} ; \boldsymbol{\mu}-$ the coefficient of consumption of nozzle opening of irrigator; $\boldsymbol{d}_{\boldsymbol{n}}$ - the diameter of nozzle opening of irrigator, $\mathrm{m}$.

The parametric dependencies of ecological efficiency and energy intensity value obtained by us allow carrying out of simulation modelling with further improvement of the process of hydrodynamic purification of exhaust gases of power plants. Simulation is carried out on basis of management of functionally independent parameters, changes of which lead to simultaneous increase of value of ecological efficiency and energy intensity value. 
Of substantial importance is the fact that management of parameters is possible on the stage of designing of the system of purification of exhaust gases of power plants. It substantially optimizes the choice of methods and means for decreasing of pollution of air medium of urban territories.

\section{Conclusions}

The solution of the actual problem of increase of ecological safety of urban territories on basis of imitational simulation of the process and system of purification of exhaust gases of power plants of housing and utilities infrastructure with the help of management of parameters substantially influencing the ecological efficiency and energy intensity value of the purification process is represented in the article.

To achieve the set goal we have:

1) built the physical model of the process of decrease of air medium pollution for the conditions of operation of power plants showing the interconnection between the main physical objects participating in this process and allowing to offer the structure of the purification system in which the pollution agents are consecutively and purposefully influenced with external impacts with the purpose of decrease of air medium pollution at the account of decrease of their stability;

2) obtained parametrical dependencies of efficiency and energy intensity value allowing to determine ways for improvement of the process of purification of exhaust gases of boiler houses with the purpose of maximally economic provision of regulatory pollution of urban territories on basis of the analysis and management of functionally independent parameters of the process, changing of which leads to simultaneous increase of values of efficiency and energy intensity value.

Practical implementation of hydrodynamic purification of exhaust gases of power plants carried out by us on basis of modelling has shown that it is impossible to offer unique solution of the purification system, everything depends on original characteristics of the facility - production and technological parameters, characteristics of technological raw materials, parameters of properties of polluting agents of exhaust gases, air medium characteristics, technological parameters of irrigation implementation, physical and chemical characteristics of irrigating fluid, parameters of exhaust source (funnel chimney).

The authors express their sincere gratitude to the staff of the Department of Environmental Engineering, Federal StateFunded Educational Institution of Higher Education Don State Technical University for advice and recommendations kindly given while writing of this article.

\section{References}

1. I. Ilchenko, News of Higher Educational Institutions. The North Caucasus region. Series: Natural sciences, 6, 79-83 (2009)
2. V. Denisov, A. Kurbatova, I. Denisova, Ecology of the city (Moscow, MarT, 2008)

3. Yu. Sedunov, S. Avdyushin, E. Borisenkov, O. Volkovitsky, N. Petrov, R. Reytenbakh, et al., Atmosphere. Reference book (reference data, models) (Leningrad, Gidrometeoizdat, 1991)

4. S. Paliwal, H. Chandra, A. Tripathi, IJMET, 4, 2-37 (2013)

5. E. Lysova, Modern situation and prospects of technical sciences development, 79-81 (2015).

6. V. Bespalov, E. Lysova, Collection of scientific papers SWorld, 2, 19-24 (2015)

7. P. Rayst, Aerosols - an introduction to the theory (Moscow, Mir, 1987)

8. H. Greene, W. Lane, Aerosols - dust, fumes and mists (Leningrad, Himiya, 1972)

9. S. Bretschneider, The Properties of Gases and Liquids, (Leningrad, Himiya, 1966)

10. P. Kouzov, L. Skryabina, Methods for determination of physical and chemical properties of industrial dusts (Leningrad, Himiya, 198).

11. N. Fuchs, The mechanics of aerosols (Moscow, Academy of Sciences Publishing House, 1955)

12. N. Strakhova, L. Ovchinnikova, A. Pleskachev, Technical solutions on protection of air environment against the polluting substances (Rostov-on-Don, Rostov State Civil Engineering University, 2002)

13. V. Bespalov, E. Lysova, O. Paramonova, L. Ganicheva, T. Pirozhnikova, A. Mishchenko, Collection of scientific papers SWorld, 3, 3-10 (2014)

14. V. Bespalov, E. Lysova, Scientific Review, 7, 914917 (2014)

15. V. Kotler, S. Belikov, Industrial heating plants: combustion of fuels and protection of the atmosphere (St Petersburg, Energotech, 2001)

16. P. Roslyakov, V. Zinina, Power systems, 12, 60-62 (1991)

17. I. Segal, Power systems, 3, 5-8 (1989)

18. E. Lysova, Yu. Eletskaya, Construction - 2003, 3133 (2003)

19. E. Lysova, L. Lisutina, Current trends in science and education, 1, 88-89 (2013)

20. V. Bespalov, S. Meshcheryakov, O. Gurova, Assessment of processes and calculation of apparatuses of environmental protection (Rostovon-Don, Rostov State Civil Engineering University, 2007)

21. V. Bespalov, N. Samarskaya, O. Paramonova, E. Lysova, Scientific Review, 9, 921-924 (2014)

22. V. Bespalov, E. Lysova, Yu. Eletskaya, Life safety. Labor and environmental protection, 7, 8-9 (2003) 